\title{
Biquadratic exchange interactions in two-dimensional magnets
}

\author{
Alexey Kartsev $\mathbb{\mathbb { D }}^{1}$, Mathias Augustin ${ }^{1}$, Richard F. L. Evans $\mathbb{\mathbb { C }}^{2}$, Kostya S. Novoselov ${ }^{3,4}$ and Elton J. G. Santos $\mathbb{\mathbb { C }}^{5 凶}$
}

Magnetism in recently discovered van der Waals materials has opened several avenues in the study of fundamental spin interactions in truly two-dimensions. A paramount question is what effect higher-order interactions beyond bilinear Heisenberg exchange have on the magnetic properties of few-atom thick compounds. Here we demonstrate that biquadratic exchange interactions, which is the simplest and most natural form of non-Heisenberg coupling, assume a key role in the magnetic properties of layered magnets. Using a combination of nonperturbative analytical techniques, non-collinear first-principles methods and classical Monte Carlo calculations that incorporate higher-order exchange, we show that several quantities including magnetic anisotropies, spin-wave gaps and topological spin-excitations are intrinsically renormalized leading to further thermal stability of the layers. We develop a spin Hamiltonian that also contains antisymmetric exchanges (e.g., Dzyaloshinskii-Moriya interactions) to successfully rationalize numerous observations, such as the non-Ising character of several compounds despite a strong magnetic anisotropy, peculiarities of the magnon spectrum of 2D magnets, and the discrepancy between measured and calculated Curie temperatures. Our results provide a theoretical framework for the exploration of different physical phenomena in 2D magnets where biquadratic exchange interactions have an important contribution.

npj Computational Materials (2020)6:150; https://doi.org/10.1038/s41524-020-00416-1

\section{INTRODUCTION}

The finding of magnetism in atomically thin van der Waals (vdW) materials $^{1,2}$ has attracted an increasing amount of interest in the investigation of magnetic phenomena at the nanoscale ${ }^{3}$. Important in the understanding and applications of 2D vdW magnets in real technologies is the elucidation of fundamental interactions that determine their magnetic properties, such as the exchange interactions between the spins. These interactions govern the magnetic ordering and can be either symmetric, which determine collinear ferromagnets and anti-ferromagnets, or antisymmetric, which promote topological non-trivial spin textures, e.g. skyrmions. Higher-order exchange terms involving the hopping of two or more electrons play a pivotal role in the spin-ordering of low-dimensional nanostructures. For instance, biquadratic (BQ) exchange interactions are critical in the elucidation of the magnetic features of several systems, such as multilayer materi$\mathrm{als}^{4}$, perovskites ${ }^{5}$, iron-based superconductors ${ }^{6,7}$, iron tellurides ${ }^{8}$ and oxides ${ }^{9}$. Indeed, in materials where the exchange is for some reason weak ${ }^{10}$ (e.g., low-temperature magnets) BQ exchange has a particularly strong influence being the case of several 2D magnets discovered so far $^{11,12}$

Here we identify that several families of $2 \mathrm{D}$ magnets including metals, insulators and small band gap semiconductors, develop substantially large BQ exchange interactions. The delicate interplay between the superexchange process through the non-magnetic atom and the Coulomb repulsion at neighboring spin-sites involving more than one electron induces sizable corrections to the total energy of the systems beyond bilinear spin models, e.g., Kitaev, Heisenberg, Ising. We developed a generalized non-Heisenberg spin Hamiltonian that includes both BQ and Dzyaloshinskii-Moriya interactions (DMI), providing a universal picture of the spin properties of $2 \mathrm{D}$ vdW magnets. We show that while $\mathrm{BQ}$ exchange interactions give substantial contributions to the thermal properties, such as in critical temperatures $\left(T_{c}\right)$, non-collinear spin effects via DMI are negligible. The model also offers insights via analytical equations into the spin excitations of $2 D$ materials as a general formalism is developed for materials that hold honeycomb crystal structure, ferromagnetism and out-of-plane easy axis in a universal basis. Our results provide the conceptual framework to understand a variety of $2 \mathrm{D}$ magnetic materials in a consistent way explaining numerous experimental observations, including controversies on the magnetic properties of $\mathrm{Crl}_{3}$ or $\mathrm{CrBr}_{3}$.

\section{RESULTS}

Biquadratic exchange interactions

In order to calculate the different exchange contributions to the total energy at the level of first-principles methods (see Supplementary Sections 1-4 for details), we map the angular dependence of the spins $\mathbf{S}_{j}=\mu_{s} \mathbf{s}_{j}$, where $\mu_{s}$ is the magnetic moment and $\left|\mathbf{s}_{j}\right|=1$, in the unit cell of the layered material ${ }^{5,13,14}$ (Fig. 1a). We rotate the spins by $\theta$ between two known spin configurations: from ferromagnetic (FM) at $\theta=0$, to antiferromagnetic (AFM) at $\theta=180^{\circ}$. Small steps in $\theta$ generate a path of quasi-continuously configurations where both energy and magnetization are allowed to relax self-consistently without any fixed constraint on the direction. The resulting curve in energy includes contributions from bilinear (BL) exchanges up to higherorder terms, e.g., BQ exchange interactions. We used different supercells that resulted in no variations of the results. We quantify the contribution of each kind of interactions using the following non-Heisenberg spin Hamiltonian:

$$
\mathcal{H}=-\sum_{i j} J_{i j}\left(\mathbf{S}_{i} \cdot \mathbf{S}_{j}\right)-\sum_{i j} \lambda_{i j} S_{i}^{z} S_{j}^{z}-\sum_{i} D_{i}\left(\mathbf{S}_{i} \cdot \mathbf{e}_{i}\right)^{2}-\sum_{i j} K_{i j}\left(\mathbf{S}_{i} \cdot \mathbf{S}_{j}\right)^{2}
$$

\footnotetext{
${ }^{1}$ School of Mathematics and Physics, Queen's University, Belfast BT7 1NN, UK. ${ }^{2}$ Department of Physics, The University of York, York YO10 5DD, UK. ${ }^{3}$ Department of Material Science \& Engineering, National University of Singapore, Block EA, 9 Engineering Drive 1, Singapore 117575, Singapore. ${ }^{4}$ Chongqing 2D Materials Institute, Liangjiang New Area, Chongqing 400714, China. ${ }^{5}$ Institute for Condensed Matter Physics and Complex Systems, School of Physics and Astronomy, The University of Edinburgh, Edinburgh EH9 3FD, UK. 凶email: esantos@ed.ac.uk
} 
a

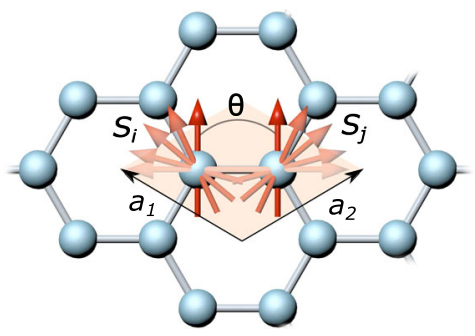

C
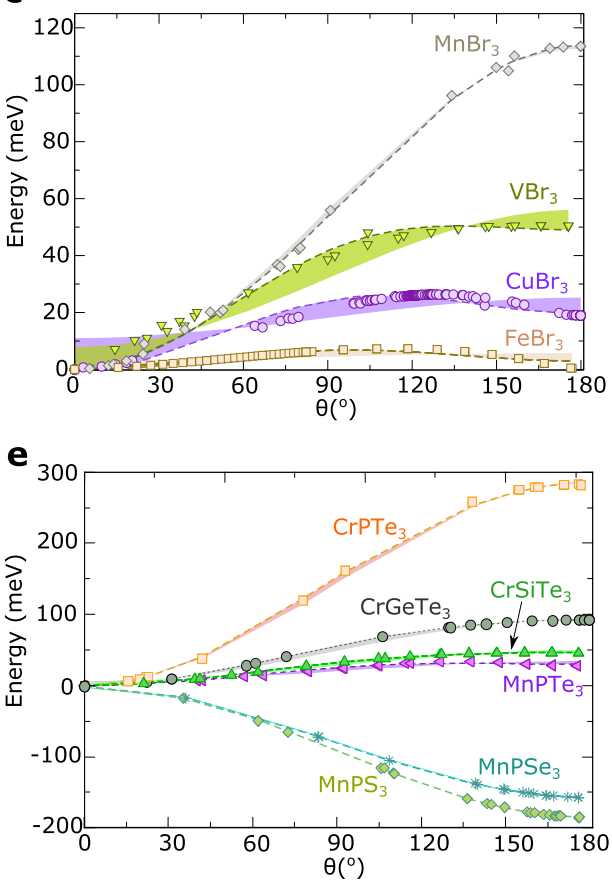

b

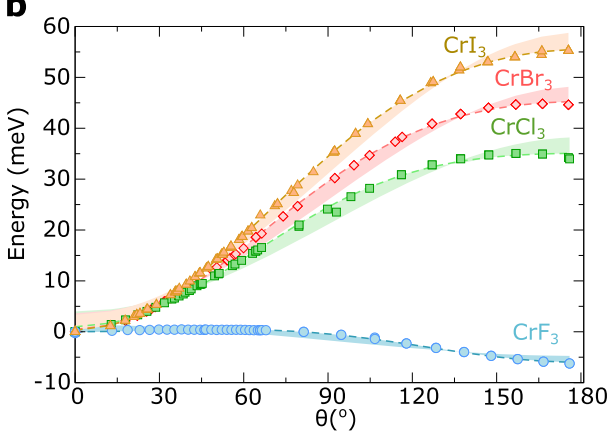

d

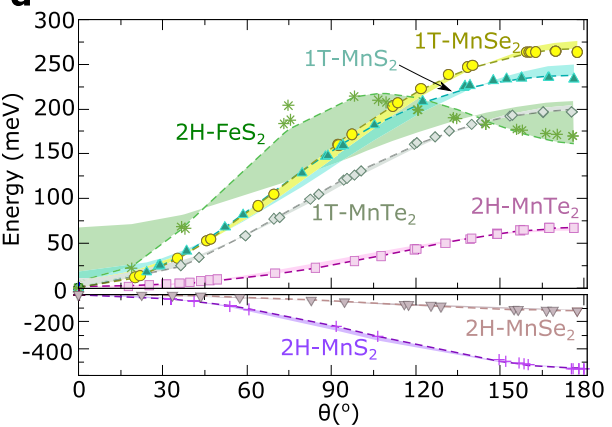

f

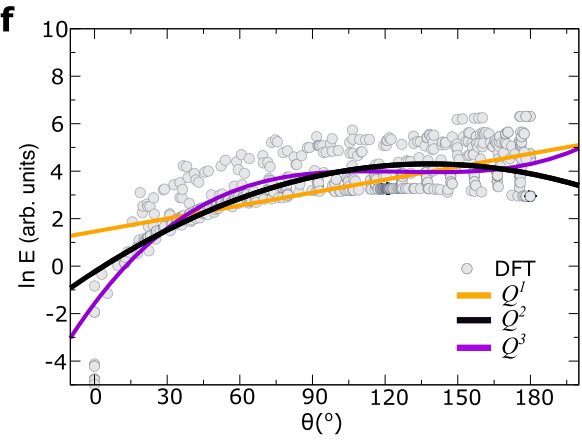

Fig. 1 Biquadratic exchange interactions in 2D magnets. a Diagram of the rotation of spins $S_{i}$ and $S_{j}$ in the unit cell (defined by vectors $\mathbf{a}_{1}$ and $\mathbf{a}_{2}$ ) of a $2 \mathrm{D}$ magnet by a relative angle $\theta$ between them. Spins are rotated symmetrically in opposite directions from $0^{\circ}$ to $180^{\circ}$. b-e Relative total energy $(\mathrm{eV})$ as a function of $\theta\left({ }^{\circ}\right)$ for different monolayers of $2 \mathrm{D}$ magnets: trihalides $(\mathrm{CrX}, \mathrm{X}=\mathrm{F}, \mathrm{Cl}, \mathrm{Br}, \mathrm{l}), \mathrm{metal}_{3}$ tribromides $\left(\mathrm{MBr}_{3}, \mathrm{M}=\mathrm{Mn}, \mathrm{Cu}, \mathrm{Fe}, \mathrm{V}\right)$, chromium based ternary tellurides $\left(\mathrm{Cr}_{2} \mathrm{X}_{2} \mathrm{Te}_{6}, \mathrm{X}=\mathrm{Ge}, \mathrm{P}, \mathrm{Si}\right)$, manganese based ternary chalcogenides $\left(\mathrm{Mn}_{2} \mathrm{P}_{2} \mathrm{X}_{6}, \mathrm{X}=\right.$ $\mathrm{S}, \mathrm{Se}, \mathrm{Te})$, transition metal dichalcogenides $\left(\mathrm{MnX}_{2}, \mathrm{X}=\mathrm{S}\right.$, Se, Te) of different phases $(2 \mathrm{H}, 1 \mathrm{~T})$ and an iron-based dichalcogenide $(2 \mathrm{H}$-FeS 2 ). The reference energy is taken at $0^{\circ}$ as the spins oriented at the same direction. Rotations can occur in-plane or out-of-plane with similar behavior. Symbols are calculated energies. Dashed lines correspond to a quadratic fitting using $E_{b q}^{\text {tot }}(\theta)=A_{0}^{b q}+A_{1}^{b q} \cdot S^{2} \cos (\theta)+A_{2}^{b q} \cdot S^{4} \cos ^{2}(\theta)$, while color filling areas indicate the deviation between the quadratic $E_{b q}^{\text {tot }}$ and a linear fitting using $E_{b l}^{\text {tot }}(\theta)=A_{0}^{b l}+A_{1}^{b l} \cdot S^{2} \cos (\theta)$. Materials that show large deviation, such as $\mathrm{CuBr}_{3}$ or $2 \mathrm{H}-\mathrm{FeS}_{2}$, develop large $\mathrm{BQ}$ exchange interactions. $\mathbf{f}$ Logarithm of the total energies (DFT) for the dataset in b-e, as a function of $\theta$. Data analytics using polynomial regression (least-squares algorithm) in terms of linear $\left(Q^{1}\right)$, quadratic $\left(Q^{2}\right)$ and cubic $\left(Q^{3}\right)$ approaches is evaluated over the calculated DFT energies (dots).

where $J_{i j}$ and $\lambda_{i j}$ are the isotropic and anisotropic BL exchanges between spins $\mathbf{S}_{i}$ and $\mathbf{S}_{j}$ on atomic sites $i$ and $j ; D_{i}$ is the on-site anisotropy with easy axis $\mathbf{e}_{i}$ and $K_{i j}$ is the BQ exchange interactions which is due to electron hopping between two adjacent $\operatorname{sites}^{15}$. We restrict the discussions to first nearestneighbor $\mathrm{BQ}$ interactions, that is, $K_{i j}=K_{b q}$, and $K_{i j}=0$ otherwise. This assumption has been shown to be sufficient to study a variety of magnetic systems with higher-order exchanges ${ }^{5,6,8,9}$. We can write Eq.(1) in a similar form separating the terms with angular and non-angular dependence as $E_{b q}^{\text {tot }}(\theta)=A_{0}^{b q}+A_{1}^{b q}$. $S^{2} \cos (\theta)+A_{2}^{b q} \cdot S^{4} \cos ^{2}(\theta)$, where $S$ is the spin moment. The different coefficients can be interpreted as the corresponding amount of $\mathrm{BL}\left(A_{1}^{b q}\right)$ and $\mathrm{BQ}\left(A_{2}^{b q}\right)$ exchanges, and the on-site energy as the spins are perpendicular to each other $\left(A_{0}^{b q}\right)$. Supplementary Section 4 gives a thorough discussion on these coefficients and how to extract analytical equations for their interpretation using
Eq. (1). We extract $A_{0}^{b q}, A_{1}^{b q}$ and $A_{2}^{b q}$ from first-principles simulations for the variation of the total energy versus $\theta$ (Supplementary Section 5).

We apply this procedure for a total of 50 compounds including the most common $2 \mathrm{D}$ magnets studied up to date including several families of trihalides $\left(\mathrm{MX}_{3}, \mathrm{M}=\mathrm{Ti}, \mathrm{V}, \mathrm{Cr}, \mathrm{Mn}, \mathrm{Fe}, \mathrm{Ni}, \mathrm{Cu} ; \mathrm{X}=\right.$ $\mathrm{F}, \mathrm{Cl}, \mathrm{Br}, \mathrm{I})$, metal tribromides $\left(\mathrm{MBr}_{3}, \mathrm{M}=\mathrm{Mn}, \mathrm{Cu}, \mathrm{Fe}, \mathrm{V}\right)$, chromium based ternary tellurides $\left(\mathrm{Cr}_{2} \mathrm{X}_{2} \mathrm{Te}_{6}, \mathrm{X}=\mathrm{Ge}, \mathrm{P}, \mathrm{Si}\right)$, metal based ternary chalcogenides $\left(M_{2} P_{2} X_{6}, M=V, C r, M n, F e, C o, N i ; X=S, S e\right.$, $\mathrm{Te})$, and transition metal dichalcogenides $\left(\mathrm{MX}_{2}, \mathrm{M}=\mathrm{Co}, \mathrm{Fe}, \mathrm{V} ; \mathrm{X}=\right.$ $\mathrm{S}, \mathrm{Se}, \mathrm{Te})$ of different phases $(2 \mathrm{H}, 1 T)$. Surprisingly, as the spins are spatially rotated away a sizable deviation from a $\cos \theta$-like behavior characteristic of BL exchange interactions (shaded area) is observed (Fig. 1b-e). The overall trend seems independent of the stoichiometric formula or the atomic elements composing the structure. The universal character of the higher-order exchange 
Table 1. Calculated biquadratic exchange $\left(K_{b q}\right)$ for several 2D magnetic materials with honeycomb (faint blue) and hexagonal (faint red) lattices using non-collinear ab initio methods as explained in the text.

\begin{tabular}{|c|c|c|c|}
\hline Material & $\mu_{M}\left(\mu_{B}\right)$ & $S$ & $K_{\mathrm{bq}}(\mathrm{meV})$ \\
\hline $\mathrm{Crl}_{3}$ & 3.36 & 1.5 & 0.21 \\
\hline $\mathrm{CrBr}_{3}$ & 3.17 & 1.5 & 0.22 \\
\hline $\mathrm{CrCl}_{3}$ & 3.05 & 1.5 & 0.21 \\
\hline $\mathrm{CrF}_{3}$ & 2.94 & 1.5 & 0.09 \\
\hline $\mathrm{MnBr}_{3}$ & 3.88 & 2 & 0.04 \\
\hline $\mathrm{CuBr}_{3}$ & 0.53 & 1 & 39.16 \\
\hline $\mathrm{FeBr}_{3}$ & 3.88 & 2 & 0.06 \\
\hline $\mathrm{VBr}_{3}$ & 1.93 & 1 & 2.85 \\
\hline $\mathrm{CrGeTe}_{3}$ & 2.81 & 1.5 & 0.35 \\
\hline $\mathrm{CrPTe}_{3}$ & 2.92 & 1.5 & 0.35 \\
\hline $\mathrm{CrSiTe}_{3}$ & 3.13 & 1.5 & 0.31 \\
\hline $\mathrm{MnPS}_{3}$ & 4.24 & 2 & 0.02 \\
\hline $\mathrm{MnPSe}_{3}$ & 4.21 & 2 & 0.02 \\
\hline $\mathrm{MnPTe}_{3}$ & 3.93 & 2 & 0.11 \\
\hline $1 \mathrm{~T}-\mathrm{MnSe}_{2}$ & 3.49 & 1.5 & 0.74 \\
\hline $2 \mathrm{H}-\mathrm{MnSe}_{2}$ & 3.49 & 2 & 0.15 \\
\hline $1 \mathrm{~T}-\mathrm{MnTe}_{2}$ & 3.71 & 2 & 0.14 \\
\hline $2 \mathrm{H}-\mathrm{MnTe}_{2}$ & 3.78 & 2 & 0.10 \\
\hline $1 \mathrm{~T}-\mathrm{MnS}_{2}$ & 3.28 & 1.5 & 1.09 \\
\hline $2 \mathrm{H}-\mathrm{MnS}_{2}$ & 3.27 & 1.5 & 1.55 \\
\hline $2 \mathrm{H}-\mathrm{FeS}_{2}$ & 2.18 & 1 & 20.11 \\
\hline
\end{tabular}

The magnitudes of the spin angular momentum (S) used in the model (Supplementary Section 4) and the magnetic moments at the metal atoms $\left(\mu_{M}\right)$ are also included.

process in layered materials can be appreciated clearer in Fig. If where data-analytics using regression algorithms (linear, quadratic, cubic) was evaluated over the computed data. The best match between regression and DFT energies is for regressions beyond linear. This suggested that even more complex magnetic interactions may take place in 2D magnetic materials such as 3-, 4spin interactions ${ }^{16,17}$, and chiral biquadratic ${ }^{18,19}$ which are not studied here. Materials with alike chemical environment (e.g., bond lengths, electron affinity, binding energy) such as $\mathrm{Crl}_{3}, \mathrm{CrBr}_{3}$ and $\mathrm{CrCl}_{3}$ present close variation of the energy and consequently similar magnitudes of the BQ exchange (Table 1). Other compounds tend to gain energy with the spin rotation and stabilize in a different magnetic coupling, for instance, $\mathrm{CrF}_{3}$ (Fig. 1b) becomes AFM ordered. This is also the case of Mn-based compounds, $2 \mathrm{H}-\mathrm{MnS}_{2}, 2 \mathrm{H}-\mathrm{MnSe}_{2}, \mathrm{MnPS}_{3}, \mathrm{MnPSe}_{3}$ (Fig. 1d, e), which agreed with magnetometry measurements ${ }^{20-22}$. It is noteworthy that $\mathrm{CuBr}_{3}$ and $2 \mathrm{H}-\mathrm{FeS}_{2}$ have substantially larger magnitudes of $\mathrm{BQ}$ exchange (Table 1) comparable to more complex materials, i.e., ferropnictides, where spin and electronic correlations are known to play a key role in the determination of their superconducting and strongly-correlated properties ${ }^{23}$. Both $\mathrm{CuBr}_{3}$ and $2 \mathrm{H}-\mathrm{FeS}_{2}$ have relatively small $\mathrm{BL}$ exchange in the range of 5.87-6.88 meV with $S=1$. For materials with small BL exchange higher-order exchange tends to be sizeable ${ }^{10}$. This result calls for further theoretical and experimental work on these two compounds whether unusual electronic interactions can be found. Furthermore, we noticed that several other compounds could not show a clear trend with $\theta$ apart from those where a specific magnetic ordering is stabilized, e.g., $\theta=0,180^{\circ}$ (Supplementary Section 6). Materials that are unable to stabilize different spin orientations are either due to strong magnetic anisotropy where a preferential spin orientation is too strong to be tilted (e.g. Ising magnets) or different spin solutions are not energetically stable ending up in non-magnetic phases ${ }^{24}$.

We have also checked whether other models can give a sound description of the magnetic properties of the quadratic dependence of the energy as a function of $\theta$. In particular, we considered two models: a Kitaev model ${ }^{25,26}$, and a Heisenberg model including biquadratic on-site magnetic anisotropy. For the latter, the magnitudes of the biquadratic on-site anisotropies are several orders of magnitude smaller than $\mathrm{BQ}$ exchange within the range 3.87-14.32 $\mu \mathrm{eV}$ (see details in Supplementary Section 7). For the former, there is no quadratic dependence on $\theta$ for the Kitaev model. We can expand the Kitaev Hamiltonian ${ }^{25}$ assuming a rotation by angle $\theta$ between the spins to show that a fitting equation of the form of $E_{\text {Kitaev }}^{\text {tot }}(\theta)=B_{0}+B_{1} \sin (2 \theta)$ can be extracted (see details in Supplementary Section 8). These results suggest that $\mathrm{BL}$ models are insufficient to describe the magnetic features of $2 \mathrm{D}$ vdW magnets.

\section{A Hubbard-based microscopic model}

To understand the microscopic mechanism of the $\mathrm{BQ}$ exchange in 2D materials, we will use the following 2D half-filled Hubbard Hamiltonian applied to the honeycomb lattice (Fig. 2a, b):

$$
H=-t_{\text {eff }} \sum_{\langle i j\rangle, \sigma} c_{i, \sigma}^{\dagger} c_{j, \sigma}+U \sum_{i} n_{i \uparrow} n_{i \downarrow}+\sum_{i, \sigma} \mu_{i, \sigma} n_{i, \sigma},
$$

where indices $i$ and $j$ denote the lattice sites, the $d$-spin states on the metal atoms $\left(\mathrm{M}_{\mathrm{A}, \mathrm{B}}\right)$ are labeled as $\sigma=\uparrow, \downarrow$, the sum $\left.<i j\right\rangle$ is over the nearest neighbors and $t_{\text {eff }}$ is the effective nearest neighbor hoping between $\mathrm{M}_{A, B}$ ions. $t_{\text {eff }}$ is due to the hybridization between $3 d^{n}$ and $n p$ orbitals $(n=2,3,4,5$ depending on the atomic element involved) at $M_{A, B}$ and $X$ atoms, respectively (Fig. $2 b$ ). The direct hopping between $\mathrm{M}_{\mathrm{A}, \mathrm{B}}$ scales with $t_{d d} \sim r^{-5}$ and therefore for relatively big distances can be neglected ${ }^{15} . U>0$ is the nonnegative on-site Coulomb repulsion, $c_{i, \sigma}^{\dagger}\left(c_{i, \sigma}\right)$ is the creation (annihilation) operator for a fermion with spin $\sigma$ at site $i$, $n=c_{i, \sigma}^{\dagger} c_{i, \sigma}$ is the density operator and $\mu_{i, \sigma}$ is the chemical potential which controls the filling of the bands. Second-order perturbation theory in Eq.(2), assuming $U>>t_{\text {eff, }}$ gives the energy contributions of the Heisenberg exchanges ${ }^{27,28}$ :

$$
\begin{aligned}
& J_{\mathrm{FM}}^{(2)}=t_{\text {eff }}^{2} /\left(U-U_{e x}\right) \\
& J_{\mathrm{AFM}}^{(2)}=t_{\text {eff }}^{2} /\left(U+U_{e x}\right) \\
& J_{\mathrm{bl}}=t_{\text {eff }}^{2} \frac{2 U_{e x}}{U^{2}-U_{e x}^{2}}
\end{aligned}
$$

where $J_{\mathrm{FM}}^{(2)}$ and $J_{\mathrm{AFM}}^{(2)}$ are the exchange energies for FM and AFM coupling at second-order, and the BL exchange is defined as $J_{\mathrm{bl}}=J_{\mathrm{FM}}^{(2)}-J_{\mathrm{AFM}}^{(2)}$. $U_{e x}$ is an energy correction for the internal spin exchange whether a spin flip is required during the hopping between $M_{A, B}$ sites (Fig. $\left.2 b\right)^{28}$. Such term can be used to stabilize or destabilize spin transfer through the $M_{A, B}-X$ covalent bonds as the exchange occurs ${ }^{15,28}$. If the electron-hopping is to an occupied orbital of the neighboring site, $U_{e x}$ will favor AFM alignment of the two spins via a superexchange interaction (see Supplementary Section 9 for details). However, if the electronhopping is to an unoccupied or a virtual state, a FM alignment will be favored by $U_{e x}{ }^{29}$. The competition between the amount of energy $U_{e x}$ to stabilize a specific coupling and the Coulomb repulsion $U$ between the electrons at an energy state may compensate each other leading to a small value of $J_{\mathrm{bl}}$. Indeed, several 2D magnets have shown low-temperature magnetism ${ }^{1,2,12}$ which is directly related with the small magnitude of the exchange interactions. In this case, it is necessary to extend the perturbation in $t_{\text {eff }} / U$ to fourth-order in Eq. (2) involving at least one electron 
a

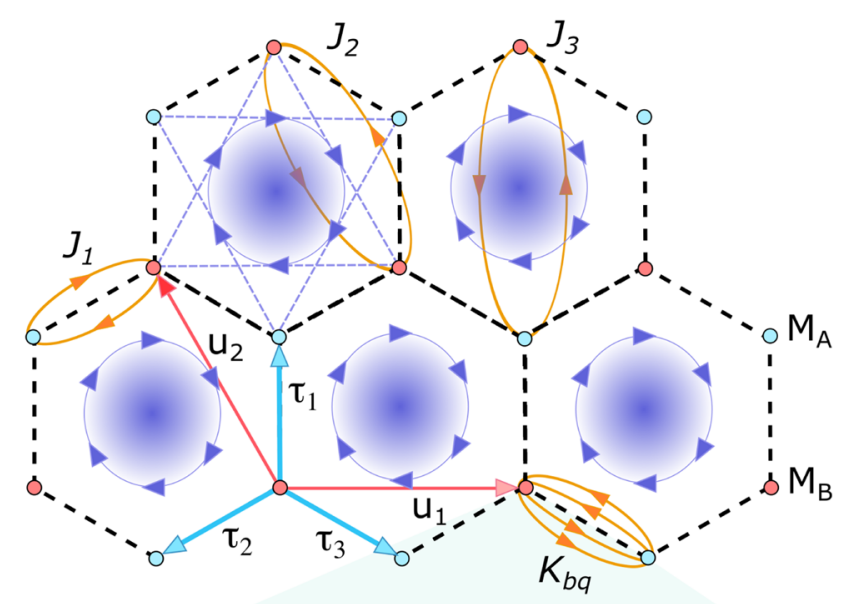

b

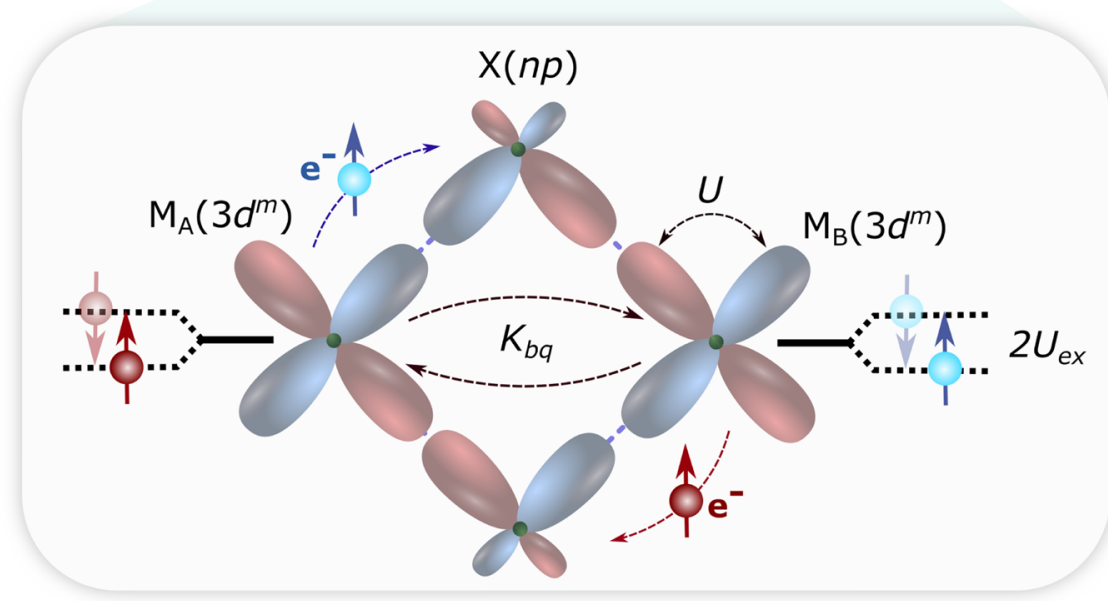

C

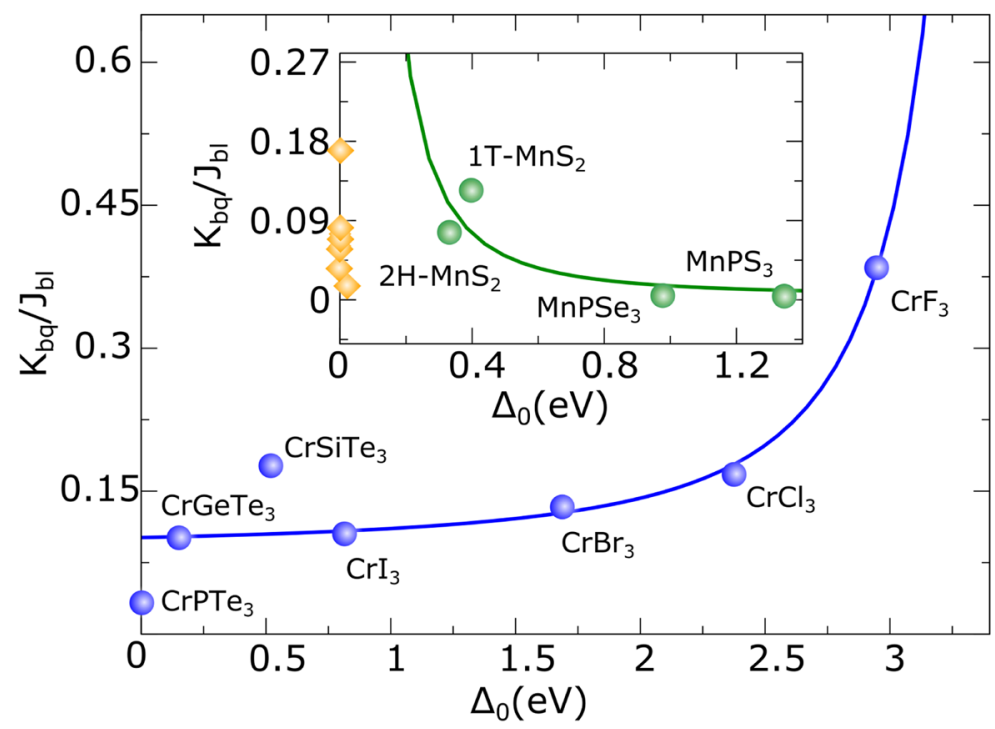


Fig. 2 Bilinear (BL) and biquadratic (BQ) exchange interactions in 2D magnets. a Schematic of the $B L$ exchanges at first $\left(J_{1}\right)$, second $\left(J_{2}\right)$ and third $\left(J_{3}\right)$ nearest neighbors $(N N)$, and BQ exchange $\left(K_{b q}\right)$ at first NN. Single and double line diagrams represent BL and BQ exchanges, respectively. The two inequivalent magnetic sites in the honeycomb lattice are shown by faint blue $\left(M_{A}\right)$ and faint red $\left(M_{B}\right)$ dots. The blue orbits inside of the hexagons represent the magnetic flux $\phi$ generated by the second-NN Dzyaloshinskii-Moriya interactions (DMI), which breaks the inversion symmetry of the lattice. The dashed lines show the magnon hopping between second NN as the magnons gain a phase given by $\phi$ (see text). The lattice vectors $\mathbf{u}_{i}(i=1,2)$ and $\tau_{j}(i=1,2,3)$ show the first and second NN on the lattice, respectively. $\mathbf{b}$ Zoom-in on the $\mathrm{BQ}$ exchange process involving two electrons between sites $\mathrm{M}_{\mathrm{A}}$ and $\mathrm{M}_{\mathrm{B}}$ with $3 d^{m}$ electrons in the valence. The $\mathrm{BQ}$ exchange $K_{b q}$ is mediated by non-magnetic atoms $\mathrm{X}$ with a valence given by $n p$ electrons, where $n$ will depend on the atomic elements involved. An on-site Hubbard $U$ term is at the $M_{A, B}$ sites with $U_{e x}$ representing the potential spin-splitting when the spins of the electrons involved in the $B Q$ exchange align ferro- or anti-ferromagnetically (Supplementary Section 4). The difference between up and down spin coupling is given by $2 U_{e x} \cdot \mathrm{c} \mathrm{K}_{b q} / J_{b l}$ versus $\Delta_{0}(\mathrm{eV})$ for all materials displaying $\mathrm{BQ}$ exchange interactions. Magnetic atoms with similar chemical environment in terms of Coulomb repulsion, exchange interactions and valence follow alike behavior for $\mathrm{K}_{b q} / \mathrm{J}_{b /}$. For instance, $\mathrm{Cr}$ in blue and $\mathrm{Mn}$ in green (inset). Orange dots show materials with dissimilar electronic configurations but with $\Delta_{0}=0$.

from both $\mathrm{M}_{\mathrm{A}, \mathrm{B}}$ sites which resulted in ${ }^{15}$ :

$$
\begin{aligned}
& J_{\mathrm{FM}}^{(4)} \propto t_{\text {eff }}^{4} /\left(U-U_{e x}\right)^{3} \\
& J_{\mathrm{AFM}}^{(4)} \propto t_{\text {eff }}^{4} /\left(U+U_{e x}\right)^{3} \\
& K_{\mathrm{bq}}=t_{\text {eff }}^{4} \frac{2\left(3 U^{2} U_{e x}+U_{e x}^{3}\right)}{\left(U^{2}-U_{e x}^{2}\right)^{3}}
\end{aligned}
$$

with $K_{\mathrm{bq}}=J_{\mathrm{FM}}^{(4)}-J_{\mathrm{AFM}}^{(4)}$ being the $\mathrm{BQ}$ exchange energy. Both Eqs. (3)-(4) show that a competition between FM and AFM couplings takes place once the electrons are hopping between different spin sites. The stabilization of one or another magnetic order is determined by several factors such as the ligand-field splitting $\Delta_{0}$ between $t_{2 g}$ and $e_{g}$ states in the metal atom in the honeycomb lattice. As the filling of both type of states determines the magnitude of the $3 d-s p$ hybridization between metals and ligands, we can approach $\Delta_{0} \approx U-U_{e x}$ being proportional to the bandgap of the material ${ }^{10}$. This condition is valid as long as the magnitudes of $U$ and $U_{e x}$ do not compete to each other. Since $U_{e x}$ is an energy correction of spin stabilization, it should comply with Hund's rule where unpaired spins occupy other states of the $3 d$ shell (Fig. 2b). It has been shown that the role of high-order exchange interactions increases on reduction of the bandgap mediated by the non-magnetic atoms which is related to the ratio of $B Q$ and $B L$ exchanges ${ }^{27,30}$. If we divide Eq. (4) and Eq. (3) and use the definition of $\Delta_{0}$, we can write a direct relationship between the exchange interactions and the bandgap for $2 \mathrm{D}$ magnets as:

$K_{\mathrm{bq}} / J_{\mathrm{bl}}=t_{\text {eff }} \frac{4 U^{2}+\Delta_{0}^{2}-2 U \Delta_{0}}{\Delta_{0}^{2}\left(2 U-\Delta_{0}\right)^{2}}$

This equation can be understood as a direct interplay between the Coulomb repulsion and the hopping of electrons between different sites subjected to the crystal field or bandgap of the material. We can consider two situations in Eq. (5): $\Delta_{0} \rightarrow 0$ and $\Delta_{0}$ $\rightarrow 2 U$ which correspond to small and large bandgap materials, respectively. This resulted in:

$$
\begin{aligned}
& K_{\mathrm{bq}} /\left.J_{\mathrm{bl}} \propto t_{\text {eff }} \frac{1}{\left(\Delta_{0}\right)^{2}}\right|_{\Delta_{0} \rightarrow 0} \\
& K_{\mathrm{bq}} /\left.J_{\mathrm{bl}} \propto t_{\text {eff }} \frac{1}{\left(2 U-\Delta_{0}\right)^{2}}\right|_{\Delta_{0} \rightarrow 2 U}
\end{aligned}
$$

Fig. 2c shows the variation of $K_{\mathrm{bq}} / J_{\mathrm{bl}}$ as a function of $\Delta_{0}$ for the core of materials displaying $\mathrm{BQ}$ exchange interactions. Strikingly, both Eqs. (6)-(7) correctly describes the overall behavior observed in our simulations. Materials with similar bonding environment, for instance, either in terms of $\mathrm{Cr}(\mathrm{Mn})$ atoms follow an increase (decrease) of $K_{\mathrm{bq}} / J_{\mathrm{bl}}$ with the bandgap, respectively. It is worth mentioning that as the compounds tend to a AFM spin alignment ${ }^{10,29}$, i.e., $\mathrm{CrF}_{3}$, they increase the value of $K_{\mathrm{bq}} / J_{\mathrm{bl}}$ being the case within the $\mathrm{Cr}$-based trihalide family $\left(\mathrm{CrX}_{3}, \mathrm{X}=\mathrm{F}, \mathrm{Cl}, \mathrm{Br}, \mathrm{I}\right)$. There is also an abrupt change in behavior for narrow bandgap materials and metals with no dependence on $\Delta_{0}$ (inset in Fig. 2c).
These results indicate that one can design the amount of $B Q$ exchange in a 2D magnet tuning its bandgap, for instance using an electric bias as recently used in $\mathrm{Crl}_{3}{ }^{31}$.

Thermal effects in 2D magnets

To verify whether $B Q$ exchange interactions will have any effect on the thermal properties of 2D vdW magnets, we have implemented Eq. (1) within the Monte Carlo Metropolis algorithm with an adaptive move ${ }^{32}$. In the spin model we assume a classical spin vector $\mathbf{S}_{i}$ on each atomic site $i$. The quantization vector for the spin is a local quantity which intrinsically includes the effects of local thermal spin fluctuations, magnon processes and spin excitations. In our implementation the $\mathrm{BQ}$ exchange is quite general and can be applied to any pair-wise exchange interaction of arbitrary range. We also consider an additional term in Eq. (1) including the local Zeeman field $\mathbf{B}$ on the magnetic ions with a length of the local atomic moment $\mu_{i}$ arising from the $\mathrm{BQ}$ exchange interactions as:

$$
\begin{aligned}
\mathcal{H}= & -\sum_{i j} J_{i j}\left(\mathbf{S}_{i} \cdot \mathbf{S}_{j}\right)-\sum_{i j} \lambda_{i j} S_{i}^{z} S_{j}^{z}-\sum_{i} D_{i}\left(\mathbf{S}_{i} \cdot \mathbf{e}_{i}\right)^{2} \\
& -\sum_{i j} K_{i j}\left(\mathbf{S}_{i} \cdot \mathbf{S}_{j}\right)^{2}-\sum_{i} \mu_{i} \mathbf{S}_{i} \cdot \mathbf{B}_{i}
\end{aligned}
$$

For atomistic spin dynamics simulations, the effective field due to the biquadratic exchange are calculated via the first-derivative of Eq. (8) on the different spin components as:

$H_{b q, i}^{\prime}=-\frac{1}{\mu_{i}} \frac{\partial \mathcal{H}}{\partial S_{i}^{\prime}}=2 K_{\mathrm{bq}} S_{j}^{\prime}\left(\mathbf{S}_{i} \cdot \mathbf{S}_{j}\right)$

where $I=x, y, z$ represents the geometrical coordinates at the atomic sites $i(j)$, and $\left(\mathbf{S}_{i} \cdot \mathbf{S}_{j}\right)=S_{i}^{x} S_{j}^{x}+S_{i}^{y} S_{j}^{y}+S_{i}^{z} S_{j}^{z}$. The effective field $H_{b q, i}^{\prime}$ therefore contributes to the total field describing the time evolution of each atomic spin using the stochastic LandauLifshitz-Gilbert equation ${ }^{33}$. We calculate up to third nearestneighbors $\lambda_{i j}$ and $J_{i j}$ in Eq. (1) on a representative set of $2 \mathrm{D}$ magnets, e.g., Cr-based trihalide family (Table 2). Supplementary Section 10 gives a thorough discussion on the calculation of the exchange parameters. Figure 3 shows the behavior of the magnetization $\left(M / M_{0}\right.$, where $M_{0}$ is the saturation magnetization at $0 \mathrm{~K})$ and the logarithm of the magnetic susceptibility $(\ln X)$ as a function of temperature $\mathrm{T}(\mathrm{K})$ for $\mathrm{Crl}_{3}, \mathrm{CrBr}_{3}$ and $\mathrm{CrCl}_{3}$. Intriguingly, the inclusion of $\mathrm{BQ}$ exchange interactions give sizable thermal effects on both $M / M_{0}$ and $\ln X$ for all materials (Supplementary

Section 11). By fitting the Monte Carlo simulations with $M(T)=$ $M_{0}\left(1-\frac{T}{T_{c}}\right)^{\beta}$ (where $\beta$ is the critical exponent) we can notice that the Curie temperatures $T_{c}$ changes by several Kelvins with the inclusion of $\mathrm{BQ}$ exchange interactions. The calculated magnitudes of $\mathrm{T}_{\mathrm{C}}$ for $\mathrm{Crl}_{3}$ and $\mathrm{CrBr}_{3}$ approach closely those measured for both compounds with almost no difference (Fig. 3a-d). The lack of experimentally measured $\mathrm{T}_{\mathrm{c}}$ for monolayer $\mathrm{CrCl}_{3}$ unable us to make a clear comparison with our simulations. The different amount of 
Table 2. Computed values of several magnetic quantities for $\mathrm{CrX}_{3}(\mathrm{X}=\mathrm{I}, \mathrm{Br}, \mathrm{Cl}, \mathrm{F})$ at different number of nearest neighbors: isotropic $\left(J_{1}, J_{2}, J_{3}\right)$ and anisotropic $\left(\lambda_{1}, \lambda_{2}, \lambda_{3}\right)$ BL exchanges.

\begin{tabular}{lccrrrr}
\hline Comp. & $J_{1}(\mathrm{meV})$ & $J_{2}(\mu \mathrm{eV})$ & $J_{3}(\mu \mathrm{eV})$ & $\lambda_{1}(\mu \mathrm{eV})$ & $\lambda_{2}(\mu \mathrm{eV})$ & $\lambda_{3}(\mu \mathrm{eV})$ \\
\hline $\mathrm{Crl}_{3}$ & 2.01 & 320.02 & 8.10 & 106.8 & -10.24 & 0.91 \\
$\mathrm{CrBr}_{3}$ & 1.66 & 164.35 & -11.60 & 20.69 & -2.06 & -0.69 \\
$\mathrm{CrCl}_{3}$ & 1.28 & 72.03 & -25.18 & 20.07 & -9.74 & -0.51 \\
$\mathrm{CrF}_{3}$ & -0.23 & 17.27 & 0.20 & 3.33 & -0.67 & -0.14 \\
\hline
\end{tabular}

The on-site magnetic anisotropy $D$ is also included. See Supplementary Section 4 for details.
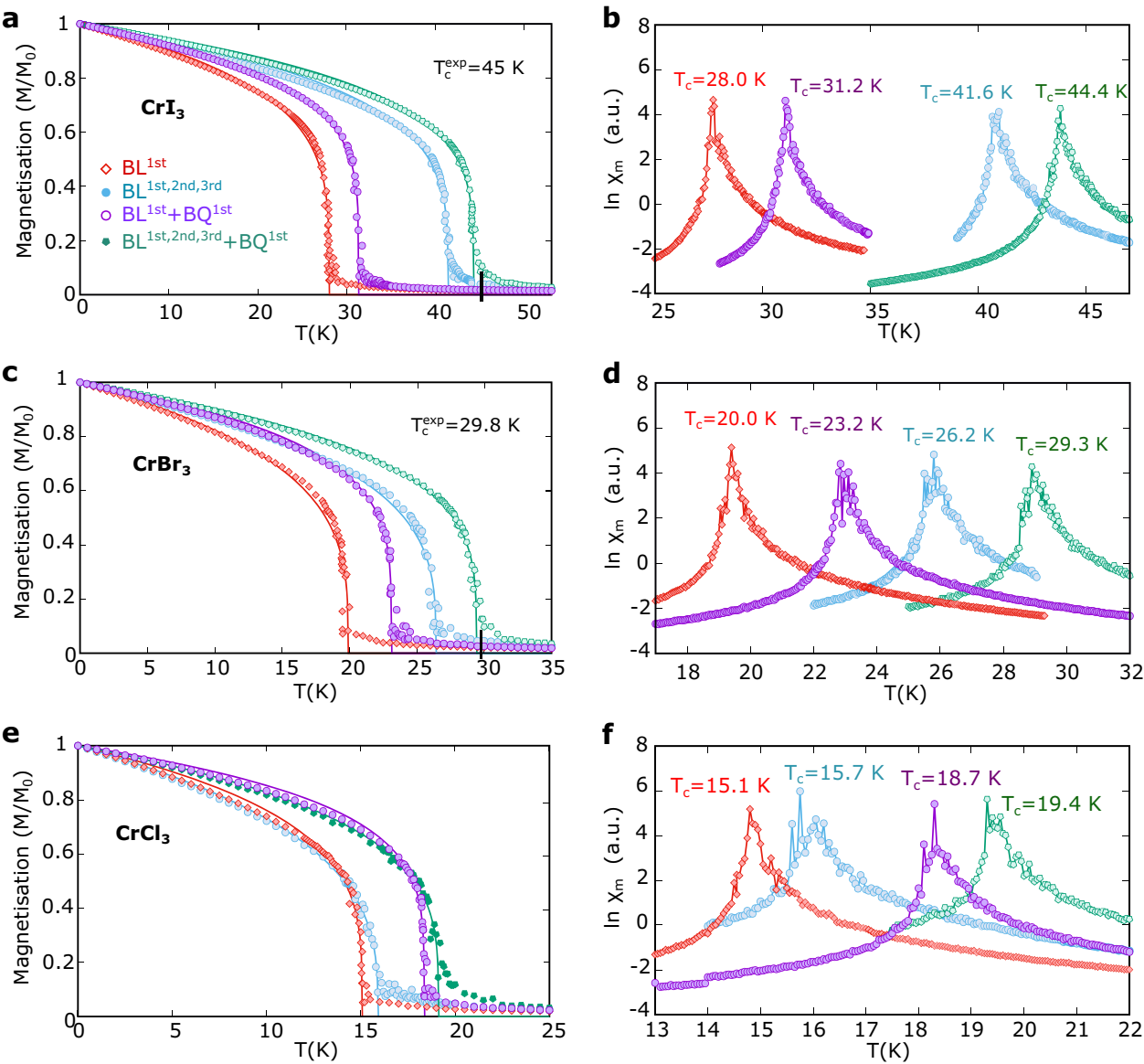

Fig. 3 Monte-Carlo simulations at different levels of theory including $B L$ and $B Q$ exchange interactions. a, $\mathbf{b}$ Magnetization (M/M $\mathrm{M}_{0}$ ) and logarithm of the magnetic longitudinal susceptibility $\ln X_{m}$ (a.u.) versus temperature (K), respectively, for monolayer $\mathrm{Crl}_{3}$. Calculated and fitting curves using $M / M_{0}=\left(1-T / T_{c}\right)^{\beta}$ (where $T_{c}$ and $\beta$ are the critical temperature and coefficient, respectively) are shown by dots and solid lines, respectively, in $\mathbf{a}$. Solid lines in $\mathbf{b}$ show the interpolation between points. Different curves correspond to different number of NN, from one up

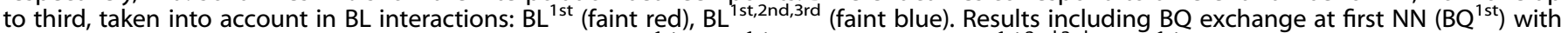
different number of $\mathrm{BL}$ exchanges are shown in purple $\left(\mathrm{BL}^{1 \mathrm{st}}+\mathrm{BQ}^{1 \mathrm{st}}\right)$ and faint green $\left(\mathrm{BL}^{1 \mathrm{st}, 2 \mathrm{nd}, 3 \mathrm{rd}}+\mathrm{BQ}^{1 \mathrm{st}}\right)$. Critical temperatures $\left(T_{\mathrm{c}}\right)$ at each level of $B L$ and $B Q$ exchange interactions are indicated at $\mathbf{b}$ with the maximum magnitude of $\ln \chi_{m}$ (a.u.) highlighted. Magnetic susceptibility is shown in logarithm scale for clarify. Experimental critical temperature $\left(T_{c}^{\mathrm{exp}}\right)$ is included for comparison. $\mathbf{c}, \mathbf{d}$ and $\mathbf{e}, \mathbf{f}$ similar plots as in $\mathbf{a}$, $\mathbf{b}$, for $\mathrm{CrBr}_{3}$ and $\mathrm{CrCl}_{3}$, respectively. Critical exponents $\beta$ extracted from the simulations for $\mathrm{BL}^{1 \mathrm{st}, 2 \mathrm{nd}, 3 \mathrm{rd}}+\mathrm{BQ}^{1 \mathrm{st}}$ are $\beta=0.22,0.24$ and 0.28 for $\mathrm{Crl}_{3}$, $\mathrm{CrBr}_{3}$ and $\mathrm{CrCl}_{3}$ respectively. Magnitudes of $\beta$ for $\mathrm{BL}^{1 \text { st,2nd,3rd }}$ are $0.25,0.28$ and 0.32 for $\mathrm{Crl}_{3}, \mathrm{CrBr}_{3}$ and $\mathrm{CrCl}_{3}$ respectively.

nearest-neighbors at BL exchanges (from 1st to 3rd) also produces substantial effects even though not enough to reproduce the experimental values of $\mathrm{T}_{c}$ for $\mathrm{Crl}_{3}$ and $\mathrm{CrBr}_{3}$. It is worth mentioning that different groups have reported distinct magnitudes of $T_{c}$ for $\mathrm{CrBr}_{3}{ }^{34-36[37-39}$, which may be due to different factors such as sample quality, defects, and doping levels. We believe that our simulations still provide an accurate picture showing the effects of the underlying exchange interactions for these low-dimensional magnets at the limit of an ideal, pristine crystal (see Supplementary
Section 12). Moreover, several other materials display larger values of $\mathrm{BQ}$ exchange interactions (Table 1) indicating that higherexchange interactions should be taken into account on the description of their magnetic properties.

Enhancement of magnetic stability

An outstanding remark on the existence of $B Q$ interactions in $2 D$ magnets is on the implications on their magnetic features. It is 
well known that several materials developed macroscopic properties due to this higher-order exchange interactions. From paramagnetic measurements of $\mathrm{Mn}^{+2}$ ions in $\mathrm{MgO}^{9}$, up to different magnetic phases in oxides ${ }^{5,13}, 40$, it is not clear whether $\mathrm{BQ}$ exchange induces any significant role effect on basic properties, such as critical temperatures or stability. To understand the intrinsic effect of higher-order exchange interactions in the thermal features of 2D magnetic materials and provide a consistent description of the underlying spin interactions, we have developed an analytical model based on spin-wave theory ${ }^{37,38}$. We generalized Eq. (1) to second order contributions on the magnetic anisotropies which resulted in:

$$
\begin{aligned}
\mathcal{H}= & -\sum_{i j} J_{i j}\left(\mathbf{S}_{i} \cdot \mathbf{S}_{j}\right)-\sum_{i j} \lambda_{i j} S_{i}^{z} S_{j}^{z}-\sum_{i} D_{i}\left(\mathbf{S}_{i} \cdot \mathbf{e}_{i}\right)^{2} \\
& -\sum_{i j} K_{i j}\left(\mathbf{S}_{i} \cdot \mathbf{S}_{j}\right)^{2}-\sum_{i} D_{i}^{b q}\left(\mathbf{S}_{i} \cdot \mathbf{e}_{i}\right)^{4}-\sum_{i j} \lambda_{i j}^{b q}\left(S_{i}^{z} S_{j}^{z}\right)^{2}
\end{aligned}
$$

where $D_{i}^{b q}$ and $\lambda_{i j}^{b q}$ are the $\mathrm{BQ}$ on-site anisotropy and $\mathrm{BQ}$ anisotropic exchange, respectively. We replace the spin operators $\mathbf{S}_{i, j}$ in Eq. (10) by bosonic creation $\left(a_{i}^{\dagger}, b_{i}^{\dagger}\right)$ and annihilation $\left(a_{i}, b_{i}\right)$ operators over the honeycomb sub-lattices $\mathcal{A}$ and $\mathcal{B}$ using Holstein-Primakoff transformations ${ }^{38}$ :

For $i \in$ Asublattice:

$$
\begin{aligned}
& S_{i}^{z}=\left(S-a_{i}^{\dagger} a_{i}\right) \\
& S_{i}^{+} \approx \sqrt{2 S} a_{i} \\
& S_{i}^{-} \approx a_{i}^{\dagger} \sqrt{2 S}
\end{aligned}
$$

Fori $\in$ Bsublattice:

$$
\begin{aligned}
& S_{i}^{z}=\left(S-b_{i}^{\dagger} b_{i}\right) \\
& S_{i}^{+} \approx \sqrt{2 S} b_{i} \\
& S_{i}^{-} \approx b_{i}^{\dagger} \sqrt{2 S}
\end{aligned}
$$

where we assume that $a_{i}^{\dagger} a_{i}<<S$ and $b_{i}^{\dagger} b_{i}<<S$ for small deviations of the spins from their ground state orientations. This gives (see Supplementary Section 13 for details):

$\tilde{\mathcal{H}}=\sum_{i}(2 \tilde{D} S+Z \cdot S(\tilde{J}+\tilde{\lambda}))\left(b_{i}^{\dagger} b_{i}+a_{i}^{\dagger} a_{i}\right)-\tilde{J} S \sum_{\langle i j\rangle}\left(a_{i}^{\dagger} b_{j}+b_{j}^{\dagger} a_{i}\right)$

where the sum over $i$ and $j$ runs over the sub-lattices and first nearest neighbors, respectively, and $Z$ is the number of first nearest-neighbors. This procedure outlines a strong implication of the inclusion of higher-order exchange interactions in the description of the magnetic properties of $2 \mathrm{D}$ magnets. That is, the enhancement of several magnetic quantities:

$\tilde{J} \approx J+2 S^{2} \cdot K_{\mathrm{bq}}$

$\tilde{\lambda} \approx \lambda+2 S^{2} \cdot \lambda^{\mathrm{bq}}$

$\tilde{D} \approx D+2 S^{2} \cdot D^{\mathrm{bq}}$

where $\lambda^{\mathrm{bq}}$ and $D^{\mathrm{bq}}$ are the $\mathrm{BQ}$ anisotropic exchanges, and the $\mathrm{BQ}$ on-site magnetic anisotropy, respectively, for first nearest neighbors. Incidentally Eqs. (12)-(14) yield several implications on the magnetic properties of the sheets, in particular, on the stabilization of magnetism in truly 2D. It is well known that in order to overcome thermal fluctuations that could destroy any magnetic order $^{39}$, sizable magnetic anisotropies need to be developed to gap the low-energy modes in the magnon spectra. That is, a spin wave gap needs to appear at the energy dispersion to act as a barrier to excitations of long-wavelength spin waves. We can show that the relation between the spin wave gap taken into account $\mathrm{BQ}$ exchange interactions $\left(\Delta_{b q}\right)$ and that at the level $\mathrm{BL}$ exchange $\left(\Delta_{b l}\right)$ for first-nearest neighbors is given by (see Supplementary
Section 13):

$\Delta_{b q}=\Delta_{b l}+4 S^{3}\left(D^{b q}+\frac{3}{2} \lambda^{b q}\right)$

where $\Delta_{b l}=2 S\left[D+\frac{Z \lambda}{2}\right]$. By using some parameters for monolayer $\mathrm{Crl}_{3}$ from Table 2, and approaching the second term in Eq. (15) as $D^{b q}+3 \lambda^{b q} \approx 10.72 \mu \mathrm{eV}$ (see Supplementary Section 7) we can estimate $\Delta_{b l}=0.81 \mathrm{meV}$ and $\Delta_{b q}=1.0 \mathrm{meV}$. The magnitude of $\Delta_{b q}$ is consistent with recent measurements of the magnon dispersion for bulk $\mathrm{Crl}_{3}$, which a spin wave gap of approximately $1.3 \mathrm{meV}$ was measured ${ }^{40}$. Furthermore, the increment of the on-site and anisotropic magnetic anisotropies (Eqs. (13)-(14)) indicates that not only spin-orbit mechanisms are behind the substantial anisotropy in $\mathrm{Crl}_{3}$ but rather higher-order exchange processes. Such BQ exchange-driven large magnetic anisotropy mechanism has been proposed for iron-based superconductors ${ }^{7,23,41}$ which successfully described their magnetic properties. A direct consequence Eqs. (12)-(14) is the increment of Curie temperatures by factor $r$ given by (see Supplementary Section 13):

$r=\frac{\tilde{T}_{C}}{T_{C}} \approx \frac{\tilde{J} \ln \left(1+2 \pi \mathrm{JS} / \Delta_{\mathrm{bl}}\right)}{\operatorname{Jn}\left(1+2 \pi \mathrm{JS} / \Delta_{\mathrm{bq}}\right)}$.

where $\tilde{T}_{C}$ and $T_{C}$ are the Curie temperatures with and without $\mathrm{BQ}$ interactions, respectively. Including few values in Eq. (16), we can roughly estimate an enhancement of $r \approx 39 \%$ for monolayer $\mathrm{Crl}_{3}$ which follows the Monte Carlo calculations (Fig. 3a, b). It is worth noticing that the model in Eq. (11), i) takes into account only firstnearest neighbors in the exchange interactions, and ii) we assume a mean-field approach in the solution of the non-linear HolsteinPrimakoff transformation (e.g. magnon-magnon interactions) to simplify the complex mathematical terms, i.e. four-operator product. Supplementary Section 14 provides a full discussion on the details involved.

Describing topological spin excitations through $\mathrm{BQ}$ and $\mathrm{DMI}$ An intriguing question that raised by the presence of $B Q$ exchange interactions is whether they play an important role in the description of magnetic quasiparticles such as magnons and non-trivial spin textures in 2D vdw magnets. It has recently been shown using neutron scattering ${ }^{40}$ that $\mathrm{Crl}_{3}$ magnet shows topological spin-excitations with two distinctive magnon bands separated by a bandgap of $4 \mathrm{meV}$ at the Dirac K-point. In spite of the clear demonstration that $\mathrm{Crl}_{3}$ can not follow an Ising model as initially pointed out ${ }^{1}$, these results indicate that non-Heisenberg interactions play an important role in the creation of spinexcitations in 2D magnetic materials. Since the gap opening at $\mathbf{K}$ is related with the inversion symmetry breaking and appearance of DMI, chirality becomes crucial in the discrimination of the magnon bound states. Moreover, it has become well established ${ }^{23,42-44}$ that isotropic spin interactions at the level of the BL Heisenberg models do not capture all features in the energy dispersion of spin-excitations in magnetic materials. There are additional contributions through uniaxial anisotropies, next-nearest neighbor interactions and the delicate balance between them, that need to be considered. In order to account for all these quantities, we extended the model in Eq. (1) with the addition of DMI:

$$
\mathcal{H}_{\text {latt }}=\mathcal{H}+\sum_{\langle\langle i j\rangle\rangle} \boldsymbol{A}_{i j} \cdot\left(\mathbf{S}_{i} \times \mathbf{S}_{j}\right)
$$

where $\boldsymbol{A}_{i j}$ is the DMI between spins $\boldsymbol{S}_{i}$ and $\boldsymbol{S}_{j}$. For a honeycomb ferromagnetic layer, with an easy axis perpendicular to the surface (z-direction), there is no breaking of the inversion symmetry of the lattice at first nearest-neighbors, e.g., $\boldsymbol{A}_{i j}^{1 \text { st }}=0$. However, contributions from the second nearest-neighbors become non-negligible as space inversion is not present. Therefore, we consider the DMI vector as $\boldsymbol{A}=v_{i j} A^{z} \boldsymbol{z}$, where $A_{z}$ is the magnitude of the DMl along of the easy-axis, and $v_{i j}= \pm 1$ represents the hopping of spins at 
second nearest-neighbors from sites $i$ to $j$ and vice-versa, respectively (Fig. 2a). Similarly as shown above, we can use Holstein-Primakoff transformations for $J_{i}>0$ to write Eq. (17) in terms of bosonic creation and annihilation operators (see details in Supplementary Section 15):

$\mathcal{H}_{\text {latt }}=\mathcal{H}_{B L}+\mathcal{H}_{B Q}+\mathcal{H}_{D M I}$

where we separate the terms due to $B L$ exchange $\left(\mathcal{H}_{B L}\right)$, from those due to $\mathrm{BQ}\left(\mathcal{H}_{B Q}\right)$ and DMI $\left(\mathcal{H}_{D M l}\right)$ which can be written as:

$\mathcal{H}_{B L}=H_{D}+\sum_{i=1}^{3} H_{i}^{\text {Isotropic }}+\sum_{i=1}^{3} H_{i}^{\text {Anisotropic }}$

$H_{B Q}=-12 K_{b q} S^{4}-2 K_{b q} S^{3}\left(\sum_{\langle i j\rangle}\left(b_{j}^{\dagger} a_{i}+a_{i}^{\dagger} b_{j}\right)-Z_{1} \sum_{i \in \mathcal{A}}^{N / 2} a_{i}^{\dagger} a_{i}-Z_{1} \sum_{i \in \mathcal{B}}^{N / 2} b_{i}^{\dagger} b_{i}\right)$

$\mathcal{H}_{D M I}=i A_{z} S\left(\sum_{\langle\langle i j\rangle\rangle \in \mathcal{A}}^{N / 2}\left(a_{i}^{\dagger} a_{j}-a_{j}^{\dagger} a_{i}\right)+\sum_{\langle\langle i j\rangle\rangle \in \mathcal{B}}^{N / 2}\left(b_{i}^{\dagger} b_{j}-b_{j}^{\dagger} b_{i}\right)\right)$

where $H_{D}$ is the on-site anisotropy term, and $H_{i}^{\text {lsotropic }}$ and $H_{i}^{\text {Anisotropic }}$ are respectively the isotropic and anisotropic parts of the $\mathrm{BL}$ exchange Hamiltonian taken into account up to third nearestneighbors $(i=1,2,3)$. The sum in $\langle i j\rangle$ runs over the first nearestneighbors at both sublattices $\mathcal{A}$ and $\mathcal{B}$ while that on $\langle\langle i j\rangle\rangle$ runs over the second nearest-neighbors specifically on either $\mathcal{A}$ or $\mathcal{B}$ lattice. We notice that the second nearest neighbors not only break the inversion symmetry of the honeycomb lattice but also generate a magnetic flux $\phi$ involving $A_{z}$ and $J_{2}$ given by:

$\phi=\tan ^{-1}\left(A_{z} / J_{2}\right)$

The magnetic flux (circular arrow) can be appreciated in Fig. 2a when the magnons (dashed lines) hop between the second nearest-neighbors. This process introduces a phase $\phi_{i j}=\mu_{i j} \phi\left(\mu_{i j}=\right.$ \pm 1 ) in the magnons as they hop from a site $i$ to $j$, and vice-versa. The different magnitudes of $\mu_{i j}$ determine whether the hopping follows the flux and consequently induces nontrivial topological properties (e.g. chirality) similarly as in the Haldane model ${ }^{45,46}$ for fermions. The topological features of the magnon bands can be described in the $\boldsymbol{k}$-space by using the Fourier transform of the creation $\left(a_{\boldsymbol{k}}^{\dagger}, b_{\boldsymbol{k}}^{\dagger}\right)$ and annihilation $\left(a_{\boldsymbol{k}}, b_{\boldsymbol{k}}\right)$ operators in Eq. (18) as:

$$
\mathcal{H}=\mathcal{H}_{0}+\sum_{\boldsymbol{k}}\left(\begin{array}{ll}
a_{\boldsymbol{k}}^{\dagger} & b_{\boldsymbol{k}}^{\dagger}
\end{array}\right)\left(\begin{array}{ll}
h_{0}(\boldsymbol{k})+h_{z}(\boldsymbol{k}) & h_{x}(\boldsymbol{k})-i h_{y}(\boldsymbol{k}) \\
h_{x}(\boldsymbol{k})+i h_{y}(\boldsymbol{k}) & h_{0}(\boldsymbol{k})-h_{z}(\boldsymbol{k})
\end{array}\right)\left(\begin{array}{l}
a_{\boldsymbol{k}} \\
b_{\boldsymbol{k}}
\end{array}\right)
$$

where the different terms can be written as (see Supplementary Section 15 for details):

$$
\begin{aligned}
\mathcal{H}_{0} & =-2 D S-3\left(J_{1}+\lambda_{1}\right) S^{2}-6\left(J_{2}+\lambda_{2}\right) S^{2}-12 K S^{4} \\
h_{0}(\boldsymbol{k}) & =\varepsilon_{0}-4 \sqrt{\left(J_{2} S\right)^{2}+\left(A_{z} S\right)^{2}} C(\boldsymbol{k}) \\
\varepsilon_{0} & =2 D S+\left(J_{1}+\lambda_{1}\right) Z_{1} S+6 K S^{3}+6\left(J_{2}+\lambda_{2}\right) S+3 J_{3} S \\
h_{x}(\boldsymbol{k}) & =-\left(J_{1}+2 K S^{2}\right) S \sum_{j=1}^{3} \cos \left(\boldsymbol{k} \cdot \boldsymbol{\tau}_{j}\right)-J_{3} S \sum_{j=1}^{3} \cos \left(\boldsymbol{k} \cdot\left(\boldsymbol{u}_{j}+\boldsymbol{\tau}_{j}\right)\right) \\
h_{y}(\boldsymbol{k}) & =-\left(J_{1}+2 K S^{2}\right) S \sum_{j=1}^{3} \sin \left(\boldsymbol{k} \cdot \boldsymbol{\tau}_{j}\right)-J_{3} S \sum_{j=1}^{3} \sin \left(\boldsymbol{k} \cdot\left(\boldsymbol{u}_{j}+\boldsymbol{\tau}_{j}\right)\right) \\
h_{z}(\boldsymbol{k}) & =4 \sqrt{\left(J_{2} S\right)^{2}+\left(A_{z} S\right)^{2}} S(\boldsymbol{k})
\end{aligned}
$$

where $C(\boldsymbol{k})=\cos (\phi) \sum_{j=1}^{3} \cos \left(\boldsymbol{k} \cdot \boldsymbol{u}_{j}\right)$ and $S(\boldsymbol{k})=\sin (\phi) \sum_{j=1}^{3}$ $\sin \left(\boldsymbol{k} \cdot \boldsymbol{u}_{j}\right)$. The vectors $\boldsymbol{\tau}_{j}$ and $\boldsymbol{u}_{j}$ are respectively between $1^{\text {st }}$ and $2^{\text {nd }}$ nearest neighbors (Fig. 2a).
The eigenvalues of Eq. (23) can be written in terms of the lower and upper energy bands as:

$$
E^{ \pm}=h_{0}(\boldsymbol{k}) \pm \sqrt{h_{x}(\boldsymbol{k})^{2}+h_{y}(\boldsymbol{k})^{2}+h_{z}(\boldsymbol{k})^{2}}
$$

Note that Eqs. (24) and (25) are general for any honeycomb material with ferromagnetic order, easy-axis perpendicular to the surface and develop DMI and $\mathrm{BQ}$ exchange interactions. For instance, we can use them to predict the energy dispersion of the magnon bands over the first Brillouin zone (BZ) of any 2D magnet, e.g., $\mathrm{Crl}_{3}$. Figure $4 \mathrm{a}$-e shows different levels of theory either using a simple XXZ model or including more sophisticated terms through the $B Q$ exchange, DMI or simultaneously all of them. It is clear that XXZ models without any contribution from DMI (Fig. $4 a-c$ ) does not describe the gap opening at the Dirac points due to the breaking of the inversion symmetry. Moreover, a model at the level of $X X Z+D M I$ as initially used to understand the magnon dispersion of $\mathrm{Crl}_{3}{ }^{40}$ does not capture entirely the full profile of the bands (Fig. $4 \mathrm{~d}$ ). The upper branch $E^{+}$becomes nearly flat with the increment of $J_{2}$ at the path $K-M-K$ while the lower magnon branch $E^{-}$turns more curved. This picture modifies substantially when $B Q$ exchange interactions are included in the $\mathrm{XXZ}+\mathrm{BQ}+\mathrm{DMI}$ model (Fig. 4e) as the magnon bands follow a similar curvature throughout the variation of $J_{2}$, although the upper branch at $\Gamma$ increased to $E_{\Gamma}^{+}=27.8 \mathrm{meV}$. A sound comparison between the $\mathrm{XXZ}+\mathrm{BQ}+\mathrm{DMI}$ model and the experimental results for $\mathrm{Crl}_{3}$ is obtained (Fig. 4f) when $J_{1}$ is varied similarly (Fig. 4a) indicating that the first nearest-neighbors are important on the stabilization of $E_{\Gamma}^{+}$. It is worth mentioning that the value of the exchange interactions taken into account in the fitting of the neutron scattering spectra ${ }^{40}$ do not separate $B L$ contributions from BQ as shown in Eqs. (12)-(14). Hence, it is not known from the fitting procedure ${ }^{40}$ what is the contribution of $K_{b q}$ to the magnon dispersion. However, such separation can be clearly stated in our model as indicated in Fig. 4f. Furthermore, even though DMI is important for the gap opening at the Dirac point, it does not contribute to the magnitudes of the magnetization or critical temperatures for any 2D magnet with an out-of-plane easy-axis and ferromagnetic aligned spins (see details in Supplementary Section 15).

\section{DISCUSSION}

In summary, we have shown the importance of biquadratic exchange interaction in the magnetic properties of $2 \mathrm{D}$ materials. We have described the phenomenology of such higher-order spin coupling, discussed its implications on several magnetic properties, and presented results at the level of non-collinear firstprinciples methods, Monte Carlo approximations and analytical models. The developed spin Hamiltonian including BQ exchange and DMI provided an accurate picture of topological spinexcitations on a generalized basis for any 2D magnet. Our results are particularly timely given the increasing interest in quantum materials, and we believe that our work will motivate the exploration of different exchange couplings and competition between critical phenomena ${ }^{47}$.

The effects of the $\mathrm{BQ}$ exchange interactions proposed here should manifest in experimentally accessible temperature range. One indication is already the accurate reproduction of experimental Curie temperatures ${ }^{1,35}$ including higher-order exchange which could not be obtained at the level of Ising, Heisenberg or Kitaev models. The magnitudes of $\mathrm{BQ}$ exchange can be in principle extracted from accurate hysteresis loops using phenomenological models ${ }^{48,49}$. Importantly in such analysis are potential temperature variations of $\mathrm{BL}$ and $\mathrm{BQ}$ exchanges with layer thickness which may indicate tunable interlayer exchanges still to be explored in 2D magnets. Frustrated 2D Heisenberg models in the presence of $\mathrm{BQ}$ exchange interactions are also a non-trivial 
a

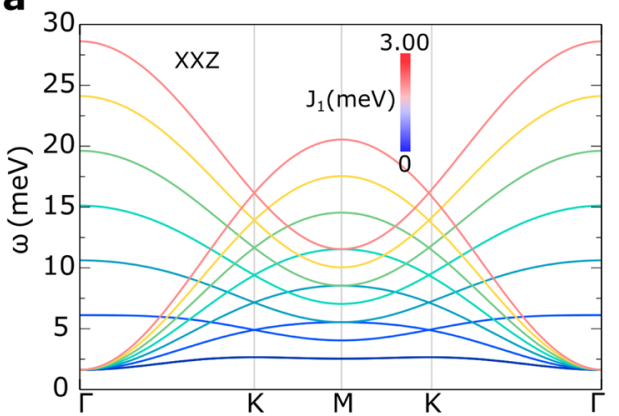

C

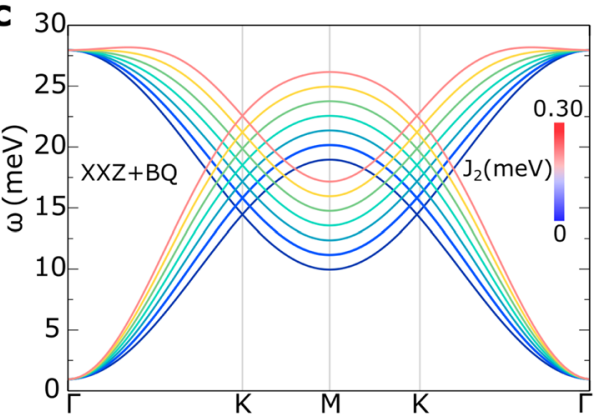

e

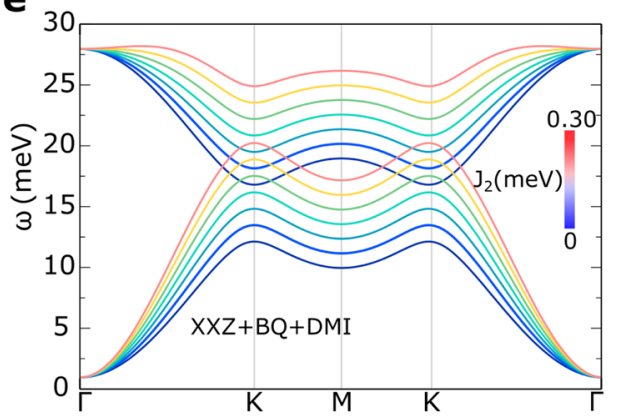

b

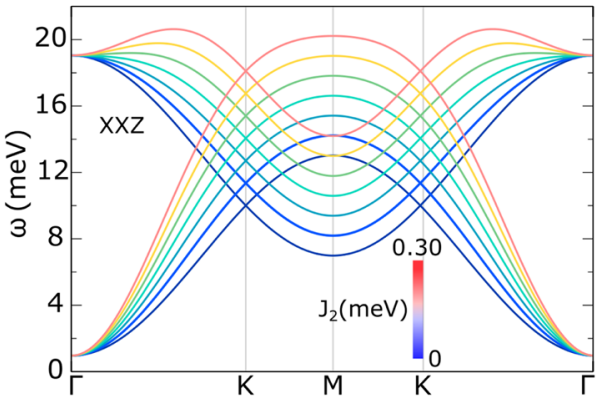

d

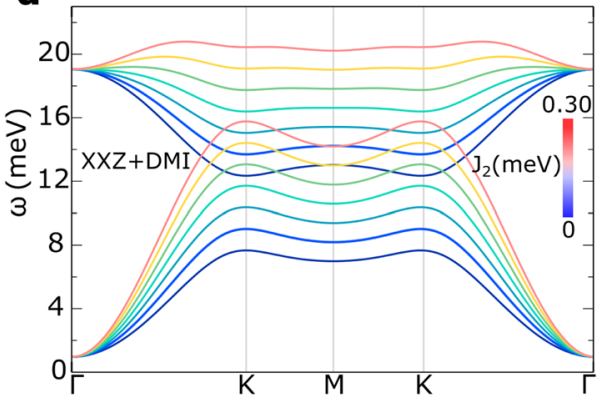

f

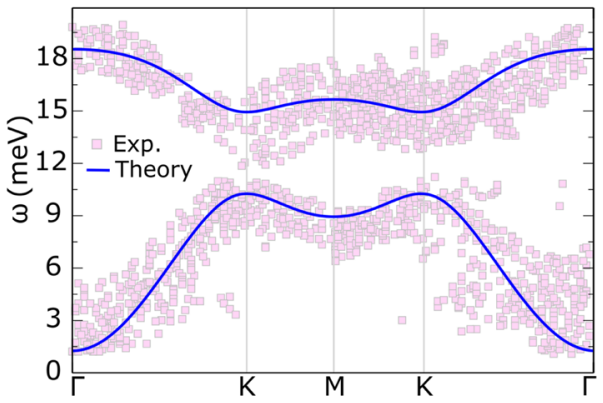

Fig. 4 Magnon spectra $\omega(\mathrm{meV})$ at different levels of theory for monolayer $\mathrm{Crl}_{3}, \mathbf{a} \omega(\mathrm{meV})$ versus $\mathbf{k}$ at the first Brillouin zone $(\Gamma-K-M-K$ $-\Gamma$ ) using a XXZ model. $J_{1}$ varies within $0-3.50 \mathrm{meV}$ in steps of $0.5 \mathrm{meV}$ from each curve (color map). $\mathbf{b}$ Similar as a, but with a fixed $J_{1}=$ $2.01 \mathrm{meV}$ and varying $J_{2}$ within $0-0.30 \mathrm{meV}$ (color map) in steps of $0.05 \mathrm{meV}$. c-e $\omega(\mathrm{meV})$ versus $\mathbf{k}$ for different models: XXZ including BQ exchange $(X X Z+B Q), X X Z$ including $\mathrm{DMI}(X X Z+D M I)$, and $X X Z$ including both $B Q$ exchange and $D M I(X X X+B Q+D M I)$. In these plots, $J_{1}=$ $2.01 \mathrm{meV}, K_{b q}=0.22$ (on c and e), $A_{z}=0.31 \mathrm{meV}^{40}$ (on $\mathbf{d}$ and e) and $J_{2}$ varies within $0-0.30 \mathrm{meV}$. $\mathbf{f}$ Comparison between the XXZ+BQ $+\mathrm{DMl}$ model and the experimental data ${ }^{40}$ recently measured for bulk $\mathrm{Crl}_{3}$. We used as parameters: $J_{1}=1.01 \mathrm{meV}, J_{2}=0.10 \mathrm{meV}, K_{b q}=0.22 \mathrm{meV}$ and $A_{z}=0.31 \mathrm{meV}^{40}$.

matter with a rich phase diagram involving incommensurate spin spirals, canted ferromagnetic states, quadrupolar phase or vertexes ${ }^{23,50-52}$. As our results indicate that $\mathrm{BQ}$ exchanges are important for 2D magnets, possible ordered and disordered magnetic regimes may be stabilized in appreciable temperatures. Moreover, it is possible to enhance or suppress BQ interactions in Mott-Hubbard systems by applying external electric fields ${ }^{53,54}$. Indeed, the control of the magnetic properties of $\mathrm{Crl}_{3}$ using electrical means has already been demonstrated ${ }^{31}$. Therefore, this opens the prospect of a coherent transfer between spin and charge degrees of freedom using short laser pulses in nanosheets.

\section{METHODS}

All methods can be found in Supplementary Materials.

\section{DATA AVAILABILITY}

The data that support the findings of this study are available within the paper and its Supplementary Information.
Received: 14 April 2020; Accepted: 9 September 2020;

Published online: 09 October 2020

\section{REFERENCES}

1. Huang, B. et al. Layer-dependent ferromagnetism in a van der waals crystal down to the monolayer limit. Nature 546, 270 -273 (2017).

2. Gong, C. et al. Discovery of intrinsic ferromagnetism in two-dimensional van der waals crystals. Nature 546, 265-269 (2017).

3. Guguchia, Z. et al. Magnetism in semiconducting molybdenum dichalcogenides. Sci. Adv 4, 1-8 (2018).

4. Slonczewski, J. C. Fluctuation mechanism for biquadratic exchange coupling in magnetic multilayers. Phys. Rev. Lett. 67, 3172-3175 (1991).

5. Fedorova, N. S., Ederer, C., Spaldin, N. A. \& Scaramucci, A. Biquadratic and ring exchange interactions in orthorhombic perovskite manganites. Phys. Rev. B 91 165122 (2015).

6. Wysocki, A. L., Belashchenko, K. D. \& Antropov, V. P. Consistent model of magnetism in ferropnictides. Nat. Phys. 7, 485(2011).

7. Zhu, H.-F. et al. Giant biquadratic interaction-induced magnetic anisotropy in the iron-based superconductor $A_{x} \mathrm{Fe}_{2-y} \mathrm{Se}_{2}$. Phys. Rev. B 93, 024511 (2016). 
8. Turner, A. M., Wang, F. \& Vishwanath, A. Kinetic magnetism and orbital order in iron telluride. Phys. Rev. B 80, 224504 (2009).

9. Harris, E. A. \& Owen, J. Biquadratic exchange between $\mathrm{mn}^{2+}$ ions in mgo. Phys. Rev. Lett. 11, 9-10 (1963).

10. Nagaev, É. L. Anomalous magnetic structures and phase transitions in nonheisenberg magnetic materials. Sov. Phys. Uspekhi 25, 31-57 (1982).

11. Gong, C. \& Zhang, X. Two-dimensional magnetic crystals and emergent heterostructure devices. Science 363, 706-717 (2019).

12. Gibertini, M., Koperski, M., Morpurgo, A. F. \& Novoselov, K. S. Magnetic 2d materials and heterostructures. Nat. Nanotechnol. 14, 408-419 (2019).

13. Hellsvik, J. et al. Tuning order-by-disorder multiferroicity in cuo by doping. Phys. Rev. B 90, 014437 (2014).

14. Novák, P., Chaplygin, I., Seifert, G., Gemming, S. \& Laskowski, R. Ab-initio calculation of exchange interactions in ymno3. Comput. Mater. Sci. 44, 79-81 (2008).

15. Takahashi, M. Half-filled hubbard model at low temperature. J. Phys. C Solid State Phys. 10, 1289-7301 (1977).

16. Krönlein, A. et al. Magnetic ground state stabilized by three-site interactions: Fe/ $\mathrm{Rh}(111)$. Phys. Rev. Lett. 120, 207202 (2018).

17. Kurz, P., Bihlmayer, G., Hirai, K. \& Blügel, S. Three-dimensional spin structure on a two-dimensional lattice: Mn/cu(111). Phys. Rev. Lett. 86, 1106-1109 (2001).

18. Brinker, S., dos Santos Dias, M. \& Lounis, S. The chiral biquadratic pair interaction. N. J. Phys. 21, 083015 (2019).

19. Grytsiuk, S. et al. Topological-chiral magnetic interactions driven by emergent orbital magnetism. Nat. Commun. 11, 511 (2020).

20. Kim, K. et al. Antiferromagnetic ordering in van der waals $2 \mathrm{~d}$ magnetic material MnPS3 probed by raman spectroscopy. 2D Mater. 6, 041001 (2019).

21. Makimura, C., Sekine, T., Tanokura, Y. \& Kurosawa, K. Raman scattering in the twodimensional antiferromagnet MnPSe3. J. Phys. Condens. Matter 5, 623-632 (1993).

22. Hastings, J. M., Elliott, N. \& Corliss, L. M. Antiferromagnetic structures of $\mathrm{mns}_{2}$, mnse $_{2}$, and mnte 2 . Phys. Rev. 115, 13-17 (1959).

23. Wysocki, A. L., Belashchenko, K. D. \& Antropov, V. P. Consistent model of magnetism in ferropnictides. Nat. Phys. 7, 485 (2011).

24. Kurz, P., Bihlmayer, G., Hirai, K. \& Blügel, S. Three-dimensional spin structure on a two-dimensional lattice: Mn /cu(111). Phys. Rev. Lett. 86, 1106-1109 (2001).

25. Kitaev, A. Anyons in an exactly solved model and beyond. Ann. Phys. 321, 2-111 (2006).

26. Xu, C., Feng, J., Xiang, H. \& Bellaiche, L. Interplay between kitaev interaction and single ion anisotropy in ferromagnetic cri 3 and crgete 3 monolayers. npj Comput. Mater. 4, 57 (2018).

27. Anderson, P. W. New approach to the theory of superexchange interactions. Phys. Rev. 115, 2 (1959).

28. Dionne, G. F. Magnetic Oxides (Springer, Boston, MA, 2009).

29. Mila, F. \& Zhang, F.-C. On the origin of biquadratic exchange in spin 1 chains. Eur. Phys. J. 16, 7-10 (2000).

30. Anderson, P. W. Theory of magnetic exchange interactions:exchange in insulators and semiconductors. (Seitz, F. \& Turnbull, D. eds.). In Solid State Physics, vol. 14, 99-214 (Academic Press, 1963).

31. Jiang, S., Shan, J. \& Mak, K. F. Electric-field switching of two-dimensional van der waals magnets. Nat. Mater. 1, 406-410 (2018).

32. Evans, R. F. et al. Atomistic spin model simulations of magnetic nanomaterials. J. Phys. Condens. Matter 26, 103202 (2014).

33. Ellis, M. O. A. et al. The landau-lifshitz equation in atomistic models. Low. Temp. Phys. 41, 705-712 (2015).

34. Kim, H. H. et al. Evolution of interlayer and intralayer magnetism in three atomically thin chromium trihalides. Proc. Natl Acad. Sci. USA 116, 11131-11136 (2019).

35. Kim, M. et al. Micromagnetometry of two-dimensional ferromagnets. Nat. Electron. 2, 457-463 (2019).

36. Zhang, Z. et al. Direct photoluminescence probing of ferromagnetism in monolayer two-dimensional crbr3. Nano Lett. 19, 3138-3142 (2019).

37. Mochizuki, M., Furukawa, N. \& Nagaosa, N. Theory of electromagnons in the multiferroic mn perovskites: The vital role of higher harmonic components of the spiral spin order. Phys. Rev. Lett. 104, 177206 (2010)

38. Dyson, F. J. General theory of spin-wave interactions. Phys. Rev. 102, 1217-1230 (1956).

39. Holstein, T. \& Primakoff, H. Field dependence of the intrinsic domain magnetization of a ferromagnet. Phys. Rev. 58, 1098-1113 (1940).

40. Mermin, N. D. \& Wagner, H. Absence of ferromagnetism or antiferromagnetism in one- or two-dimensional isotropic heisenberg models. Phys. Rev. Lett. 17, 1133-1136 (1966)

41. Chen, L. et al. Topological spin excitations in honeycomb ferromagnet $\mathrm{Cri}_{3}$. Phys. Rev. X 8, 041028 (2018).
42. Hu, J., Xu, B., Liu, W., Hao, N.-N. \& Wang, Y. Unified minimum effective model of magnetic462 properties of iron-based superconductors. Phys. Rev. B 85, 144403 (2012).

43. Elliott, R. J. \& Thorpe, M. F. The effects of magnon-magnon interaction on the two-magnon spectra of antiferromagnets. J. Phys. C Solid State Phys. 2, 1630-1643 (1969).

44. Nauciel-Bloch, M., Sarma, G. \& Castets, A. Spin-one heisenberg ferromagnet in the presence of biquadratic exchange. Phys. Rev. B 5, 4603-4609 (1972).

45. Coldea, R. et al. Spin waves and electronic interactions in $\mathrm{la}_{2} \mathrm{CuO}_{4}$. Phys. Rev. Lett. 86, 5377-5380 (2001).

46. Haldane, F. D. M. Model for a quantum hall effect without landau levels: Condensed-matter realization of the "parity anomaly". Phys. Rev. Lett. 61, 2015-2018 (1988)

47. Owerre, S. A. Topological honeycomb magnon hall effect: a calculation of thermal hall conductivity of magnetic spin excitations. J. Appl. Phys. 120, 043903 (2016).

48. Basov, D. N., Averitt, R. D. \& Hsieh, D. Towards properties on demand in quantum materials. Nat. Mater. 16, 1077-1088 (2017).

49. Strijkers, G. J., Kohlhepp, J. T., Swagten, H. J. M. \& de Jonge, W. J. M. Origin of biquadratic exchange in Fe/Si/Fe. Phys. Rev. Lett. 84, 1812-1815 (2000).

50. Fullerton, E. E. \& Bader, S. D. Temperature-dependent biquadratic coupling in antiferromagnetically coupled fe/fesi multilayers. Phys. Rev. B 53, 5112-5115 (1996).

51. Hayden, L. X., Kaplan, T. A. \& Mahanti, S. D. Frustrated classical heisenberg and $x y$ models in two dimensions with nearest-neighbor biquadratic exchange: exact solution for the ground-state phase diagram. Phys. Rev. Lett. 105, 047203 (2010).

52. Kaplan, T. Frustrated classical heisenberg model in one dimension with nearestneighbor biquadratic exchange: Exact solution for the ground-state phase diagram. Phys. Rev. B 80, 012407 (2009).

53. Läuchli, A., Mila, F. \& Penc, K. Quadrupolar phases of the $s=1$ bilinear-biquadratic heisenberg model on the triangular lattice. Phys. Rev. Lett. 97, 087205 (2006).

54. Barbeau, M. M. S., Eckstein, M., Katsnelson, M. I. \& Mentink, J. H. Optical control of competing exchange interactions and coherent spin-charge coupling in twoorbital Mott insulators. SciPost Phys. 6, 27 (2019).

\section{ACKNOWLEDGEMENTS}

EJGS acknowledges computational resources through the UK Materials and Molecular Modelling Hub for access to THOMAS supercluster, which is partially funded by EPSRC (EP/P020194/1); CIRRUS Tier-2 HPC Service (ec131 Cirrus Project) at http:// www.cirrus.ac.uk EPCC funded by the University of Edinburgh and EPSRC (EP/ P020267/1); ARCHER UK National Supercomputing Service (http://www.archer.ac.uk) via Project d429. EJGS acknowledges the EPSRC Early Career Fellowship (EP/T021578/ 1) and the University of Edinburgh for funding support.

\section{AUTHOR CONTRIBUTIONS}

E.J.G.S. conceived the idea, supervised the project and undertook the analysis. A.K. performed the first-principles and Monte Carlo simulations under the supervision of E.J.G.S. R.F.L.E. implemented the biquadratic exchange interactions in the Monte Carlo algorithm. M.A., A.K. and E.J.G.S. developed the analytical and numerical models used to describe the biquadratic exchange and DMI. K.S.N. contributed on the discussions and significance of the results. E.J.G.S. wrote the paper with inputs from all authors. All authors contributed to this work, read the manuscript, discussed the results, and agreed to the contents of the manuscript.

\section{COMPETING INTERESTS}

The authors declare no conflict of interests.

\section{ADDITIONAL INFORMATION}

Supplementary information is available for this paper at https://doi.org/10.1038/ s41524-020-00416-1.

Correspondence and requests for materials should be addressed to E.J.G.S.

Reprints and permission information is available at http://www.nature.com/ reprints

Publisher's note Springer Nature remains neutral with regard to jurisdictional claims in published maps and institutional affiliations. 
Open Access This article is licensed under a Creative Commons Attribution 4.0 International License, which permits use, sharing, adaptation, distribution and reproduction in any medium or format, as long as you give appropriate credit to the original author(s) and the source, provide a link to the Creative Commons license, and indicate if changes were made. The images or other third party material in this article are included in the article's Creative Commons license, unless indicated otherwise in a credit line to the material. If material is not included in the article's Creative Commons license and your intended use is not permitted by statutory regulation or exceeds the permitted use, you will need to obtain permission directly from the copyright holder. To view a copy of this license, visit http://creativecommons. org/licenses/by/4.0/.

(c) The Author(s) 2020 